\title{
AVERSÃO ÀS PERDAS NO COMPORTAMENTO DO CONSUMIDOR E A DINÂMICA DE CURTO PRAZO DOS PREÇOS DAS HABITAÇÕES
}

\author{
Luis Guilherme Bernardes* \\ Joaquim Montezuma de Carvalho**
}

\begin{abstract}
Este artigo incorpora o fenómeno de aversão às perdas, observado no comportamento dos consumidores, no estudo da dinâmica de curto prazo dos preços das habitações. Os resultados obtidos vêm ao encontro da literatura existente sobre a eficiência do mercado habitacional, que sugere que os "booms" verificados nos preços das habitações resultam de expectativas míopes em relação à evolução desses preços, mais do que de alterações significativas nos fundamentais do mercado. O contributo principal deste artigo é o de propor uma explicação para o que, na ausência de choques importantes nos fundamentais, desencadeia este comportamento miope e consequente evolução irracional dos preços. A nossa conclusão essencial é que a psicologia dos investidores assume um papel fundamental mesmo na fase inicial dos ciclos habitacionais. Especificamente, a aversão às perdas no comportamento dos consumidores significa que uma simples inversão no ciclo de rendimento pode ser suficiente para iniciar ou terminar um periodo de rápida expansão dos preços das habitações. Evidência empírica obtida no mercado habitacional de Lisboa é consistente com esta
\end{abstract}

* Professor Auxiliar, Instituto Universitário de Desenvolvimento e Promoção Social, Universidade Católica Portuguesa, Pólo de Viseu do Centro Regional das Beiras.

** Assistente, Instituto Universitário de Desenvolvimento e Promoção Social, Universidade Católica Portuguesa, Pólo de Viseu do Centro Regional das Beiras. 
hipótese e sugere a importância de testes quantitativos deste efeito a um nível não agregado.

Palavras-chave: Mercado de habitação, eficiência, aversão às perdas, comportamento, consumo.

\section{INTRODUÇÃO}

Este artigo incorpora o fenómeno psicológico de aversão às perdas, observado no comportamento dos consumidores, no estudo da dinâmica de curto prazo dos preços das habitações. Os resultados obtidos vêm ao encontro da literatura existente sobre a eficiência do mercado habitacional, que sugere que os booms verificados nos preços das habitações resultam de expectativas míopes em relação à evolução desses preços, mais do que de alterações significativas nos fundamentais do mercado (rendimento, stock habitacional, taxa de juro ou número de famílias). O contributo principal deste artigo é o de propor uma explicação para o que, na ausência de choques importantes nos fundamentais, desencadeia este comportamento míope e consequente evolução irracional dos preços das habitações - uma questão tanto mais importante quanto se conhece a inércia comportamental normalmente associada ao consumo de bens duráveis ${ }^{1}$. A nossa conclusão essencial é que a psicologia dos investidores assume um papel relevante, mesmo na fase inicial do ciclo habitacional. Especificamente, a aversão às perdas no comportamento dos consumidores significa que uma simples inversão do ciclo de rendimento pode ser suficiente para iniciar ou terminar um período de rápida expansão dos preços das habitações.

Vários estudos têm vindo a confirmar uma assimetria fundamental nos padrões de consumo dos indivíduos que viola a hipótese do rendimento permanente. De acordo com estes estudos, quando existe alguma incerteza, os agentes económicos mostram-se menos propensos à diminuição do consumo em resposta a más notícias acerca do seu rendimento futuro, do que ao aumento do consumo em resposta a boas notícias. Vários autores, como Shea (1995), Bateman et al. (1997) e Bowman et al. (1999), atribuem esta resposta assimétrica a alterações previsíveis do rendimento ao fenómeno de reference-dependence e, em particular, à aversão às perdas. Reference-dependence (ou a dependência das preferências relativamente a uma referência) implica que o bem estar dos agentes depende, não só, do seu consumo presente, mas também da 
forma como esse consumo presente se relaciona com um dado valor de referência, normalmente o nível anterior ou habitual de consumo. A aversão às perdas, por seu lado, estabelece que o desagrado provocado por uma perda é muito superior à satisfação proporcionada pelo ganho equivalente.

A necessidade de tomar como ponto de referência o nível de consumo recente significa que os estudos atrás mencionados incidem especialmente sobre o consumo (regular) de bens não duráveis (e serviços, no caso de Bowman et al.). Neste artigo, contudo, defendemos que tal comportamento assimétrico pode também influenciar a procura habitacional. De facto, Bowman et al. (1999) observam que a assimetria no comportamento do consumidor é menos robusta quando avaliada em termos do consumo total. Uma vez que os indivíduos podem escolher entre consumir bens duráveis e não duráveis (limitados, naturalmente, pela restrição orçamental), isso poderá significar que a resistência exibida pelos indivíduos à diminuição do consumo de não duráveis em resposta a uma previsível perda no rendimento é parcialmente compensada pela diminuição das suas despesas em bens duráveis (e logo em habitação) relativamente ao planeado ${ }^{2}$.

Para formalizar este argumento, utilizamos um simples modelo de preferências reference-dependent com aversão às perdas, em todo idêntico ao proposto por Bowman et al (1999), com excepção da introdução de uma variável relativa ao consumo habitacional, entendido aqui como uma medida do stock habitacional desejado pelos indivíduos e, logo, da procura de habitações. Em consonância com o implícito nos estudos de Shea (1995) e Bowman et al. (1999), o pressuposto de partida para o suporte teórico do nosso argumento é o de que a utilidade do consumo habitacional deriva, quase exclusivamente, do valor absoluto desse consumo, dada a natureza durável do bem. Por outras palavras, consideramos que o ganho de utilidade de curto prazo que resulta exclusivamente do facto de se ter adquirido uma casa melhor que a anterior é pouco relevante no contexto da satisfação global proporcionada pelo bem (até porque a motivação para aquisição de casa nem sempre é o seu usufruto). Tendo em conta este pressuposto, a maximização da função de utilidade de um agente representativo desta economia permitenos chegar a algumas conclusões importantes. Se ignorarmos mudanças no ponto de referência e assumirmos a não existência de aversão às perdas, continuamos a verificar uma relação positiva, relativamente bem definida, entre o rendimento real per capita e a procura habitacional aquilo a que chamaremos o efeito-standard de rendimento. Contudo, 
quando analisado o efeito de possíveis alterações nos pontos de referência e a existência de aversão às perdas, verificamos que essa relação entre o rendimento e a procura habitacional se pode modificar substancialmente, em especial durante ciclos negativos de rendimento. A consequência é a seguinte assimetria: enquanto durante ciclos económicos positivos o efeito-standard de rendimento tenderá a prevalecer, durante ciclos negativos a procura de habitações deverá cair para além do previsto por esse efeito-standard, implicando também uma quebra desproporcional nos preços das habitações ${ }^{3}$.

Com o objectivo de empiricamente testarmos estas hipóteses, socorremo-nos do modelo stock-flow tradicional (tipo DiPasquale e Wheaton, 1996) de determinação dos preços das habitações. O modelo stock-flow assume que, no curto prazo, os preços das habitações se ajustam rapidamente de forma a equilibrar a procura ao valor existente do stock imobiliário, dado o lento ajustamento deste último. Os dados utilizados dizem respeito ao mercado habitacional do Distrito de Lisboa para um período de 10 anos (de 1988 a 1997), tendo sido recolhidas observações trimestrais ${ }^{4}$. A série adoptada, apesar de algumas limitações, exibe também algumas vantagens importantes para o estudo em causa. A saber, durante o período de tempo referido, para além de terem ocorrido dois ciclos completos de rendimento, o mercado habitacional de Lisboa sofreu um crescimento sem restrições de solo significativas, as taxas de juro praticadas no crédito à habitação diminuíram de valores acima dos $18 \%$ para valores perto dos $5 \%$ e a competição entre as instituições bancárias, no contexto da desregulamentação do sistema financeiro, tornaram as restrições à concessão de crédito hipotecário relativamente pouco sensíveis ao ciclo económico ${ }^{5}$. Por outro lado, durante este período não ocorreram importantes choques externos, salvo a tendência de diminuição da dimensão dos agregados familiares e a introdução de um novo referencial de juros (a taxa LISBOR) na segunda metade de 1996, aspectos que não ignoramos nos nossos testes.

Os resultados obtidos são estatisticamente bastante robustos e consistentes com o previsto pelo nosso modelo. Quando estimamos o modelo stock-flow tradicional, encontramos a já esperada relação positiva e significativa entre o rendimento real per capita e os preços das habitações. Contudo, como anteriormente sugerido, esta formulação tradicional esconde alguns aspectos importantes relacionados com a dinâmica de curto prazo dos preços das habitações, visíveis apenas quando "partimos" a amostra de acordo com o sinal dos ciclos de rendimento. Neste caso, as nossas estimações revelam uma assimetria 
clara na evolução dos preços das habitações em função do ciclo económico. Quando o rendimento sobe, os preços das habitações convergem, no essencial, para o valor implícito no efeito-standard de rendimento. Contudo, quando o rendimento desce, verificamos que os preços das habitações caiem substancialmente abaixo do previsto por esse efeito. Para além disso, e de acordo com a restante literatura, observamos que os preços das habitações são uma função negativa do stock habitacional e do custo de posse esperado - o que nós designaremos como "motivo especulativo" - assumindo, neste último caso, que os indivíduos adoptam expectativas adaptativas (ou míopes) para o cálculo da apreciação futura dos preços.

Estes resultados sugerem duas questões fundamentais. Em primeiro lugar, que significado têm em termos da dinâmica de curto prazo os preços das habitações? Em segundo lugar, serão estes resultados uma consequência, de facto, da aversão às perdas no comportamento dos consumidores?

Relativamente à dinâmica dos preços das habitações, os nossos resultados apresentam algumas implicações importantes, que desenvolvemos mais à frente. Grande parte dos modelos tradicionais depende da interrupção do comportamento míope ou de alterações relativamente significativas nos fundamentais do mercado para justificar um novo ciclo de preços habitacionais. As nossas conclusões determinam que, devido à aversão às perdas nas preferências dos consumidores, uma simples inversão do ciclo de rendimento pode ser suficiente para desencadear ou terminar um período de rápido crescimento dos preços. Especificamente, após um ciclo negativo de rendimento, logo que o seu valor exiba sinais de retoma, a pressão negativa sobre a procura habitacional que resulta da aversão às perdas desaparece, permitindo que os preços cresçam de forma desproporcional relativamente ao previsto pelo efeito-standard de rendimento. Este crescimento repentino dos preços irá sustentar o processo especulativo que então se inicia, o qual será catalisado pela rigidez da oferta. Inversamente, quando o rendimento per capita começa a diminuir, a aversão às perdas desencadeia uma redução dos preços que é, uma vez mais, desproporcional. A dinâmica do período de contracção que se segue é, depois, o inverso do observado durante o período de expansão. Mais à frente, ilustramos estas conclusões, simulando as consequências de simples inversões do ciclo de rendimento sobre o mercado habitacional. Os resultados dessa simulação confirmam a extrema volatilidade a que os preços das habitações estão 
sujeitos devido a esta assimetria, mesmo na ausência de choques relevantes nos fundamentais.

Além disso, os nossos resultados reforçam as conclusões de estudos recentes de Genesove e Mayer $(2000,2001)$, que investigaram a aversão às perdas no comportamento dos vendedores e concluíram que, em períodos de contraç̧ão do mercado, o preço mínimo a que estes estão dispostos a vender (preço de reserva) é menos flexível que o dos compradores. De acordo com estes autores, esta é a razão principal para duas características atípicas do mercado habitacional: a forte correlação positiva entre os preços das habitações e o volume de transacções; e a correlação negativa entre os preços praticados e o tempo que decorre até à venda efectiva. A aversão às perdas no comportamento dos consumidores é consistente com estas características, reforçando aliás o seu significado, uma vez que alarga a discrepância entre o preço de reserva dos vendedores e o preço a que os compradores estão dispostos a comprar, durante os períodos de recessão.

Finalmente, a falta de dados adequados impossibilita um teste mais concreto da verdadeira importância da aversão às perdas no contexto dos nossos resultados empíricos. Assim sendo, fica em aberto a possibilidade dos resultados obtidos não serem fundamentalmente devidos à aversão às perdas. Mais adiante neste artigo, discutimos, com base em testes adicionais e na literatura sobre o comportamento dos consumidores e sobre o mercado habitacional, algumas explicações alternativas para a assimetria evidenciada nos nossos testes. A nossa conclusão é que o fenómeno de aversão às perdas é, de facto, uma razão essencial para os resultados empíricos obtidos, embora reconheçamos a necessidade de mais testes empregando, de preferência, dados não agregados.

Este artigo tem mais 4 secções. A secção 2 desenvolve o modelo teórico e analisa as suas implicações. A secção 3 testa empiricamente essas implicações e a secção 4 apresenta uma breve discussão dos resultados e respectivas limitações. A secção 5 resume as principais conclusões.

\section{O MODELO}

Para representar os fenómenos de reference-dependence e aversão às perdas nas preferências dos consumidores, propomos a seguir uma função de utilidade idêntica à especificada por Bowman et al. (1999). A 
única diferença significativa é que consideramos que, para além do consumo de não duráveis, a utilidade do indivíduo depende também do consumo do bem habitacional. Relativamente aos não duráveis assumimos, tal como Bowman et al. (1999), que a utilidade dos agentes depende não apenas do nível absoluto do consumo presente mas também da forma como esse consumo presente se compara com um determinado nível de referência, neste caso o valor do consumo passado. No que concerne à habitação, e tal como foi explicado na introdução, assumimos o caso extremo em que a utilidade do agente é uma função estrita do valor absoluto do seu consumo, devido à natureza durável deste bem. Para além disso, ignoramos neste enquadramento teórico as questões de inércia comportamental já atrás referidas, assim como as motivações de ordem especulativa para a procura de habitações. Desta forma, podemos representar a função de utilidade dos agentes económicos, num determinado período, através da função $U(r, c, h)$, tal que:

$$
U(r, c, h)=(w(r)+v(c-r), h)
$$

onde $c$ mede o nível de consumo presente de bens não duráveis, $r$ é o nível de referência para o consumo desses bens e $h$ representa o consumo presente do bem habitacional. Adicionalmente, $v($.$) é uma função de$ utilidade tipo "ganhos-perdas" que representa a forma como os agentes são afectados por desvios no consumo de bens não duráveis relativamente ao seu nível de referência e $w($.) pode ser definida como a utilidade de referência. À semelhança de Bowman et al. (1999), consideramos que a função de utilidade "ganhos-perdas" é independente do nível de referência em si mesmo e que $w($.$) capta qualquer efeito residual que$ alterações no nível de referência possam ter na utilidade dos indivíduos, assumindo $c-r$ constante. Como é hábito, consideramos que a função $U(., .,$.$) é definida para todos os valores não-negativos de r, c$ e $h$, e que a função $v($.) é normalizada de maneira a tomar o valor zero quando $c=r$, de forma que $v(0)=0$ e $U(r, r, h)=(w(r), h)$. Por último, estabelecemos que todos os componentes da função de utilidade são contínuos, têm um declive finito e são duplamente diferenciáveis, excepto quando $c=r$ : uma consequência da aversão às perdas, como explicaremos mais à frente. 


\subsection{A Função de Utilidade "Ganhos-Perdas"}

No âmbito deste artigo, o aspecto principal da função de utilidade definida em (1) é a função "ganhos-perdas" $v($.$) . De acordo com o$ trabalho referência de Kahneman e Tversky (1979) e estudos subsequentes sobre este assunto ${ }^{6}$, esta função "ganhos-perdas" (ou "value function", segundo a "Prospect Theory" de Kahneman e Tversky, 1979) é caracterizada por três princípios essenciais, resumidos a seguir nas hipóteses A1, A2 e A3:

A1. $v(x)$ é estritamente crescente em $x$.

A2. $v(x)+v(-x)<0$ para $x>0$, e $v(y)+v(-y)<v(x)+v(-x)$ se $y>x>0$.

A3. $v(x)$ é estritamente côncavo para $x>0$ e estritamente convexo para $x<0$.

A hipótese A1 significa simplesmente que $U(r, c, h)$ é crescente em $c$. A hipótese $\mathrm{A} 2$ implica que os indivíduos dão maior relevo a uma possível perda do que a um ganho equivalente e que a desutilidade marginal associada a uma perda é superior à utilidade marginal obtida a partir de um ganho equivalente. Finalmente, A3 traduz a diminuição da nossa sensibilidade marginal aos ganhos e perdas, à medida que eles vão aumentando em valor. Por outras palavras, A3 significa que as nossas percepções subjectivas são uma função côncava da amplitude das variações relativamente ao nosso ponto de referência, donde resulta que os indivíduos são avessos ao risco quando se trata de analisar potenciais ganhos, mas amantes do risco quando se trata de avaliar potenciais perdas.

Neste artigo, estamos especialmente interessados no fenómeno de aversão às perdas, o qual é captado pela hipótese A2. Esta hipótese é em grande parte partilhada pelos modelos tradicionais de utilidade em que esta é representada por uma função côncava, à qual está associada o conceito de aversão ao risco. O que diferencia a aversão às perdas da aversão ao risco é o excessivo desagrado dos indivíduos relativamente às perdas, mesmo quando as perdas e ganhos envolvidos são bastante pequenos - um resultado justificado, por exemplo, pelo chamado efeito-posse: o valor associado à perda de um bem é maior que o valor associado ao ganho desse mesmo bem quando não está ainda na nossa posse ${ }^{7}$. Esta ideia é formalizada na hipótese A4: 
A4. Se $x>0$, então $\lim _{x \rightarrow 0} \frac{v^{\prime}(-x)}{v^{\prime}(x)} \equiv L>1$.

\subsection{Reference-Dependence, Aversão às Perdas e a Procura Habitacional}

Uma análise completa da forma como estes atributos do comportamento individual afectam a procura no mercado de habitação é bastante difícil, dado o pouco conhecimento existente relativamente à dinâmica conjunta de $w($.$) e v($.) e acerca da formação dos pontos de referência dos indivíduos. Assim, qualquer conclusão genérica sobre este assunto estaria sempre bastante dependente da imposição de hipóteses algo fortes. Tendo em conta esta premissa, o objectivo desta secção é apenas o de ilustrar o tipo de consequências que a reference-dependence e a aversão às perdas podem ter sobre a dinâmica de curto prazo da procura habitacional. Neste sentido, o que propomos a seguir são hipóteses meramente simplificadoras destinadas a isolar os efeitos destes fenómenos comportamentais neste contexto e, em especial, a proporcionar um suporte algébrico relativamente simples à intuição que sustenta o nosso argumento.

O primeiro passo nesta abordagem simplificada passa por propor uma forma particular (e familiar) de $U(r, c, h)$ que tenha em consideração as hipóteses anteriores, A1 a A4. Assim, consideramos que a função de utilidade $U(r, c, h)$ é definida pela seguinte condição:

$$
U(r, c, h)=\left\{\begin{array}{l}
\left(r^{\beta}+(c-r)^{\rho}\right) h^{1-\beta}, \text { para } c \geq r \\
\left(r^{\beta}-K[-(c-r)]^{\rho}\right) h^{1-\beta}, \text { para } c<r
\end{array}\right.
$$

onde $K>1$ representa o coeficiente de aversão às perdas dos indivíduos (ou seja, a intensidade com que os indivíduos sobreavaliam as perdas relativamente a ganhos equivalentes) e $\rho, \beta<1$.

Como já foi referido, um aspecto sobre o qual a literatura é ainda inconclusiva é o da formação dos pontos de referência por parte dos indivíduos. A hipótese tradicional é a de que o ponto de referência actual é uma função simultânea do consumo realizado no último período e do ponto de referência adoptado nesse mesmo período. Ou seja: 


$$
r_{t}=\alpha c_{t-1}+(1-\alpha) r_{t-1}
$$

onde $\alpha \in[0,1]$ representa a velocidade com que os indivíduos ajustam o seu ponto de referência em resposta ao consumo recente. No nosso caso, de forma a manter a análise relativamente simples, assumimos que os indivíduos ajustam totalmente o seu ponto de referência ao nível de consumo no período anterior: ou seja $\alpha=1 \mathrm{e}$, assim, $r_{t}=c_{t-1}$. À frente, concluímos que relaxar este pressuposto não afecta de forma significativa os nossos principais resultados.

Por último, e de acordo com a literatura na área comportamental, assumimos que os indivíduos ignoram a forma como as suas decisões de consumo hoje vão afectar o ponto de referência e, logo, a sua utilidade, amanhã $\tilde{}^{8}$. Mais ainda, de acordo com os argumentos propostos por Shea (1995) e Bowman et al. (1999), consideramos que os agentes formulam as suas decisões de consumo para o período $t$ no início desse período e que essas decisões são baseadas nas expectativas dos agentes relativamente ao seu rendimento durante o período (incluindo, por isso, informação acerca de possíveis alterações no valor do rendimento num futuro próximo). Desta forma, num determinado período $t$, a solução do problema de maximização de utilidade de um agente representativo desta economia é dada por:

$$
\left.\begin{array}{l}
h_{t}=\frac{p_{t}(1-\beta)}{\rho}\left(r_{t}^{\beta}\left(c_{t}-r_{t}\right)^{1-\rho}+\left(c_{t}-r_{t}\right)\right) \\
c_{t}=\frac{Y_{t}}{p_{t}}-\frac{(1-\beta)}{\rho}\left(r_{t}^{\beta}\left(c_{t}-r_{t}\right)^{1-\rho}+\left(c_{t}-r_{t}\right)\right)
\end{array}\right\}, \operatorname{para} c_{t}>r_{t}
$$

e

$$
\left.\begin{array}{l}
h_{t}=\frac{p_{t}(1-\beta)}{K \rho}\left(r_{t}^{\beta}\left[-\left(c_{t}-r_{t}\right)\right]^{1-\rho}-K\left[-\left(c_{t}-r_{t}\right)\right]\right) \\
c_{t}=\frac{Y_{t}}{p_{t}}-\frac{(1-\beta)}{K \rho}\left(r_{t}^{\beta}\left[-\left(c_{t}-r_{t}\right)\right]^{1-\rho}-K\left[-\left(c_{t}-r_{t}\right)\right]\right)
\end{array}\right\}, \text { para } c_{t}<r_{t}
$$


onde $p_{t}$ é o preço dos bens não duráveis no período $t$ (que assumimos como um dado neste modelo) e $Y_{t}$ é o rendimento nominal esperado para o mesmo período.

A partir destas condições, e dadas as nossas hipóteses anteriores, podemos derivar uma série de proposições explicativas da forma como alterações no rendimento e no ponto de referência influenciam a dinâmica de curto prazo da procura habitacional ${ }^{9}$.

Proposição 1: Se ignorarmos o efeito de aversão às perdas e possiveis alterações no ponto de referência, concluímos que a procura do bem habitacional é uma função crescente, embora não totalmente bem definida, do nivel do rendimento real per capita.

O que a Proposição 1 define é aquilo que atrás chamámos efeito-standard de rendimento. Como seria de esperar, a relação entre a procura de habitações e o nível do rendimento exibe um valor claramente positivo. Apesar de tudo, esta relação não é tão bem definida como aquela proposta no modelo tradicional. De facto, a presença de um ponto de referência na utilidade do indivíduo relativa a bens não duráveis significa que $\partial h_{t} / \partial Y_{t}$ é uma função desse ponto de referência e, também, uma função decrescente do nível de rendimento real - uma tendência que é, aliás, observada em diversos estudos ${ }^{10}$. As proposições seguintes mostram que a natureza desta relação será ainda mais distorcida à medida que considerarmos a evolução do ponto de referência e o fenómeno de aversão às perdas.

Proposição 2: Se Al a A4 se verificarem e $r_{t}=c_{t-1}$, então $h_{t}<h_{t-1}$ para $Y_{t}=Y_{t-1}$.

Esta proposição descreve o impacte base que uma alteração no nível de referência do indivíduo pode ter nas suas decisões de consumo aquilo que designaremos como o efeito ajustamento-da-referência. A conclusão é que, se houver um rápido ajustamento do ponto de referência por parte dos indivíduos, a resistência a um decréscimo no consumo de não duráveis será intensificada, mesmo se à custa de uma menor despesa em duráveis. Este trade-off adquire ainda maior relevo durante ciclos negativos de rendimento, como resulta da Proposição 3. 
Proposição 3: Se Al a A4 se verificarem e $Y_{t}=Y_{t-1}$, então $\left.h_{t}\right|_{c_{t}<r_{t}}<\left.h_{t}\right|_{c_{t}>r_{t}}$ desde que o coeficiente de aversão às perdas (K) não seja muito pequeno.

A Proposição 3 implica que, quando a quebra no rendimento é tal que o consumo de não duráveis se torna inferior ao ponto de referência, o impacte negativo sobre o consumo de duráveis é, com grande probabilidade, potenciado pelo fenómeno de aversão às perdas.

Em temos da evolução de curto prazo da procura habitacional e, portanto, dos preços das habitações, as consequências destas proposições são sintetizadas nos Corolários 1 e 2, que a seguir apresentamos. Estes corolários pretendem ser mais genéricos do que as proposições atrás apresentadas e tomam em conta a possibilidade de $\alpha<1$, o que diminuiria a robustez da Proposição 2.

Corolário 1: Durante um ciclo positivo de rendimento (tal que, $\left.Y_{t}>Y_{t-1}\right)$, a relação entre a procura de habitações e o rendimento deverá exibir uma tendência semelhante àquela implícita no efeito-standard de rendimento. Contudo, de acordo com a Proposição 2, se os sucessivos aumentos no nivel de rendimento forem suficientemente pequenos é possível que a procura de habitações se situe ligeiramente abaixo do previsto por esse efeito.

Corolário 2: Durante um ciclo negativo de rendimento (tal que, $\left.Y_{t}<Y_{t-1}\right), \quad$ a procura de habitações cairá como resultado do efeito-standard de rendimento. Contudo, com base na Proposição 3, se a quebra no rendimento for suficientemente grande, o grau de diminuição da procura ultrapassará aquele previsto por esse efeito.

Em síntese, os Corolários 1 e 2 demonstram a assimetria de que temos vindo a falar. Durante ciclos positivos de rendimento, a procura habitacional será determinada por efeitos algo contraditórios: aqueles expressos nas Proposições 1 e 2, ainda que o segundo possa ser relativamente pouco significativo. Durante ciclos negativos, este conflito desaparece. Uma diminuição do rendimento combina, pelo menos, dois efeitos negativos sobre a procura habitacional: o efeito-standard de rendimento e o efeito ajustamento-da-referência, devendo mesmo acumular um terceiro efeito: a aversão às perdas. 


\section{ANÁLISE EMPÍRICA}

O modelo usado para formalizar e testar os resultados propostos na secção anterior é um modelo stock-flow tipo-DiPasquale e Wheaton (1996). A equação que, segundo a teoria stock-flow, traduz o preço de equilíbrio no mercado de habitação pode ser escrita da seguinte forma:

$$
P_{t}=\alpha_{0}+\alpha_{1} y_{t}+\alpha_{2} U_{t}+\alpha_{3} s_{t}
$$

onde $P_{t}$ é o índice de preços das habitações no período $t, y_{t}$ é a média do rendimento real por família (a que iremos chamar rendimento real per capita) no mesmo período, $U_{t}$ é o custo de posse esperado pelo comprador (taxa de juro menos apreciação/depreciação do valor do imóvel) e $s_{t}$ é o stock habitacional por agregado familiar. As hipóteses deste modelo estabelecem que $\alpha_{1}$ é positivo enquanto $\alpha_{2}$ e $\alpha_{3}$ são negativos.

Apesar das suas limitações-nomeadamente, a abordagem de equilíbrio parcial (a oferta é tida como estática no curto prazo) - esta equação capta de forma resumida as relações principais que determinam a dinâmica de curto prazo dos preços das habitações. Aquilo que propomos a seguir é uma extensão deste modelo que nos permitirá testar a validade das nossas hipóteses. Especificamente, a equação básica que propomos é dada por:

$$
P_{t}=\beta_{o}+\beta_{1} y_{t}+\beta_{2} \text { Rise } * \Delta y_{t}+\beta_{3} \text { Fall } * \Delta y_{t}+\beta_{4} U_{t}+\beta_{5} s_{t}+\varepsilon
$$

onde $\Delta y_{t}=y_{t}-y_{t-1}$, Rise é uma variável dummy igual a 1 quando $\Delta y_{t}>0$ e igual a 0 nos restantes casos, e Fall é uma variável dummy igual a 1 quando $\Delta y_{t}<0$ e igual a 0 nos restantes casos. Tendo em conta os argumentos propostos na secção 2 , as hipóteses nulas que a seguir testamos são:

- $\beta_{1}>0$ reflectindo a natureza prociclíca da procura, demonstrada no efeito-standard de rendimento e definida na Proposição 1 . A robustez estatística deste coeficiente é difícil de avaliar, em virtude da natureza não-linear da relação que derivámos entre o rendimento per capita e a procura de habitações. 
- $\beta_{2} \leq 0$ como consequência da Proposição 2. Contudo, o significado estatístico deste coeficiente é questionável, dada a fragilidade teórica da Proposição 2 (vide pressupostos relativos à velocidade de ajustamento do ponto de referência).

- $\quad \beta_{3}>0$ como resultado das Proposições 2 e 3. Desta vez, tendo em conta que a combinação de efeitos é inequívoca, esperamos um coeficiente estatisticamente robusto.

Finalmente, e de acordo com o modelo stock-flow tradicional, esperamos obter $\beta_{4}<0$ e $\beta_{5}<0$, ambos estatisticamente significativos.

\subsection{Dados}

Tal como referimos na introdução deste artigo, os dados dizem respeito ao Distrito de Lisboa, para o período compreendido entre 1988 e 1997 (ver anexo estatístico para uma descrição das variáveis e para fontes e critérios usados). Relativamente às variáveis utilizadas existem alguns aspectos prévios que convém esclarecer. Em primeiro lugar, e à semelhança dos testes empíricos realizados por DiPasquale e Wheaton (1996), devido à falta de dados disponíveis para a variável "número de famílias", utilizamos o número de empregados como estimativa da evolução dessa variável ${ }^{11}$. Em segundo lugar, e de acordo com a literatura existente, assumimos que os indivíduos exibem expectativas adaptativas relativamente à taxa de valorização dos preços das habitações. Em particular, consideramos que $U_{t}$ é uma função da taxa de juro e de uma média aritmética simples das taxas anuais (nominais) de valorização dos preços das habitações observadas nos quatro últimos trimestres - um horizonte temporal que se baseia nalgumas conclusões recentes sobre a formação dos pontos de referência, que revelam uma sensibilidade especial dos indivíduos à informação recebida durante o último ano (ver, por exemplo, Heath et al., 1999) ${ }^{12}$. Também foram testados outros horizontes temporais mas as implicações sobre os resultados foram mínimas.

As vantagens principais associadas ao uso destes dados foram já referidas na introdução deste artigo. Os vários ciclos de rendimento, a amplitude da variação das taxas de juro, o crescimento sem restrições do stock habitacional e a ausência de choques externos relevantes deverão contribuir para a natureza generalizável dos nossos resultados. As desvantagens, contudo, são também relevantes. No entanto, para além de 
algumas insuficiências estatísticas (mencionadas no anexo estatístico) e da curta dimensão temporal da série usada, os principais problemas encontrados são comuns aos dos estudos que baseiam as suas conclusões em dados agregados e em índices de preços. Em todo o caso, os nossos resultados empíricos não evidenciam qualquer desvio nítido em relação ao enfatizado na teoria ou aos resultados obtidos em estudos anteriores que utilizaram a metodologia de DiPasquale e Wheaton (1996). Mais importante, a robustez das nossas estimações é bastante alta, sugerindo que os nossos resultados são pelo menos qualitativamente significativos mesmo que os parâmetros não traduzam de forma precisa a dimensão das relações calculadas.

\subsection{Resultados}

Os resultados da estimação feita, usando o Método dos Mínimos Quadrados, são apresentados na tabela 1 .

Tabela 1

DETERMINANTES DOS PREÇOS DAS HABITAÇÕES

\begin{tabular}{rrrr}
\hline \hline Variáveis & \multicolumn{1}{c}{$(1)$} & \multicolumn{1}{c}{$(2)$} & \multicolumn{1}{c}{$(3)$} \\
\hline Cons_ & $238,7^{* *}$ & $238,4^{* *}$ & $247,4^{* *}$ \\
& $(20,1)$ & $(20,7)$ & $(24,3)$ \\
$y_{t}$ & $8,74^{*}$ & $9,06^{*}$ & 5,36 \\
& $(1,81)$ & $(1,93)$ & $(1,32)$ \\
$S_{t}$ & $-35,5^{* *}$ & $-35,5^{* *}$ & $-34,2^{* *}$ \\
& $(-6,60)$ & $(-6,87)$ & $(-7,68)$ \\
$U_{t}$ & $-18,6^{* *}$ & $-25,3^{* *}$ & $-26,1^{* *}$ \\
& $(-2,11)$ & $(-2,77)$ & $(-3,49)$ \\
Rise * $\Delta y_{t}$ & & $-15,04$ & $-11,38$ \\
& & $(-1,04)$ & $(-1,24)$ \\
Fall $* \Delta y_{t}$ & & $33,7^{*}$ & $48,9^{* *}$ \\
& & $(2,19)$ & $(4,05)$ \\
\hline Obsv. & 35 & 35 & 35 \\
\hline$R^{2}$ & 0,87 & 0,89 & 0,92 \\
\hline \hline
\end{tabular}

Notas: A variável dependente é o índice de preços das habitações para o Distrito de Lisboa (ver anexo estatístico para mais detalhes); t-estatísticas em parêntesis.

* significativo a $10 \% ; * *$ significativo a $5 \%$. 
Na coluna (1) testamos o modelo stock-flow tradicional, tal como está formulado na equação (6). Os resultados obtidos são os esperados, revelando uma relação positiva entre o rendimento per capita $\left(y_{t}\right)$ e os preços das habitações $\left(P_{t}\right)$, e uma forte correlação negativa entre $P_{t}$ e as outras duas variáveis explicativas: o custo de posse $\left(U_{t}\right)$ e o stock habitacional $\left(s_{t}\right)$. As colunas (2) e (3) refinam esta análise (e questionam algumas das suas conclusões) ao incluir informação sobre os ciclos económicos de curto prazo. A coluna (2) assume que os indivíduos formulam as suas decisões de consumo apenas com base em informação muito recente; em particular, assumimos que $\Delta y_{t}$ reflecte unicamente uma comparação entre o rendimento do trimestre actual e o do trimestre anterior. Já a coluna (3) considera que os indivíduos comparam o seu rendimento actual com o rendimento obtido durante os últimos quatro trimestres - uma hipótese provavelmente mais correcta, tal como foi explicado anteriormente ${ }^{13}$. Os resultados em ambas as regressões são bastante semelhantes apesar de, tal como esperado, serem ligeiramente mais robustos no cenário usado na coluna (3). Tais resultados permitem-nos apontar duas conclusões principais, ambas consistentes com as nossas hipóteses.

Primeiro, encontramos uma clara relação positiva, apesar de pouco significativa na coluna (3), entre o rendimento real per capita e os preços das habitações. O declive estimado - o qual pode ser interpretado como o efeito-standard de rendimento, uma vez que ignora os efeitos específicos dos ciclos de curto prazo - é dado por $\beta_{1}$. De acordo com o seu valor, um aumento de $1 \%$ no rendimento real per capita provoca, em termos médios, um aumento de $0,32 \%$ no índice de preços das habitações $(0,19 \%$ na regressão 3). Quanto à reduzida robustez estatística desta relação, ela não deixa de ser consistente com os nossos argumentos. De facto, de acordo com a nossa Proposição 1, a simples existência de preferências reference-dependent no domínio dos bens não duráveis implica uma relação entre o rendimento e os preços das habitações que, apesar de claramente procíclica, é insuficientemente definida. Uma outra explicação, mais convencional, é o lento ajustamento (ou inércia comportamental) a que normalmente estão sujeitas as despesas em bens duráveis ${ }^{14}$. Aliás, estes aspectos assumem um relevo maior no nosso caso, em virtude da reduzida dimensão da amostra investigada. 
A segunda conclusão decorre da observação dos coeficientes estimados para as variáveis Rise $* \Delta y_{t}$ e Fall $* \Delta y_{t}$, os quais confirmam uma assimetria importante. Durante os ciclos positivos de rendimento, os preços das habitações tendem, no essencial, para o valor implícito no efeito-standard de rendimento, uma vez que a correlação estimada entre o aumento do rendimento e os preços das habitações (medida por Rise $* \Delta y_{t}$ ) é estatisticamente não significativa. $\mathrm{O}$ valor negativo de $\beta_{2}$ é consistente com os argumentos apresentados, nomeadamente com o efeito ajustamento-da-referência apresentado na Proposição 2. Já no que respeita aos ciclos negativos de rendimento, verificamos um desvio evidente dos preços em relação ao previsto pelo efeito-standard de rendimento, uma vez que o coeficiente de Fall $* \Delta y_{t}$ é superior a zero e estatisticamente bastante robusto. Em particular, concluímos que durante ciclos negativos de rendimento uma diminuição de $1 \%$ no rendimento leva, em média, a uma diminuição entre $1,4 \%$ e $1,8 \%$ nos preços das habitações, dependendo da especificação utilizada. Estes valores de elasticidade superiores a 1 sugerem um ajustamento do consumo do bem habitação, face a uma redução do rendimento, bastante superior ao verificado no consumo de bens não duráveis, sendo por isso consistentes com o argumento baseado na aversão às perdas.

Por último, realizámos alguns testes de robustez a estas conclusões, impondo algumas ligeiras alterações às especificações do modelo. Os principais resultados obtidos são em tudo idênticos aos anteriores, reforçando pois a validade das nossas hipóteses. Os valores apresentados na Tabela 2 comprovam este facto para o cenário comportamental assumido na regressão (3) da Tabela 1. 
Tabela 2

DETERMINANTES DOS PREÇOS DAS HABITAÇÕES (TESTES DE ROBUSTEZ)

\begin{tabular}{rrrrr}
\hline \hline Variáveis & \multicolumn{1}{c}{$(1)$} & \multicolumn{1}{c}{$(2)$} & \multicolumn{1}{c}{$(3)$} & \multicolumn{1}{c}{$(4)$} \\
\hline Cons_ & $248,9^{* *}$ & $239,3^{* *}$ & $251,6^{* *}$ & $248,1^{* *}$ \\
& $(24,6)$ & $(21,8)$ & $(26,3)$ & $(23,6)$ \\
$y_{t}$ & 4,99 & 21,37 & 4,07 & 0,49 \\
& $(1,23)$ & $(1,34)$ & $(1,00)$ & $(0,15)$ \\
$S_{t}$ & $-33,9^{* *}$ & $-34,2^{* *}$ & $-34,1^{* *}$ & $-30,3^{* *}$ \\
& $(-7,66)$ & $(-7,77)$ & $(-7,73)$ & $(-7,22)$ \\
$U_{t}$ & $-26,7^{* *}$ & $-26,5^{* *}$ & $-24,7^{* *}$ & $-25,3^{* *}$ \\
& $(-3,56)$ & $(-3,55)$ & $(-3,60)$ & $(-3,26)$ \\
Rise * $\Delta_{t}$ & $-0,41$ & $-11,51$ & $-9,05$ & $-6,65$ \\
& $(-1,18)$ & $(-1,26)$ & $(-1,00)$ & $(-0,72)$ \\
Fall $* \Delta y_{t}$ & $1,85^{* *}$ & $48,56^{* *}$ & $48,85^{* *}$ & $49,76^{* *}$ \\
& $(4,09)$ & $(4,01)$ & $(4,09)$ & $(4,68)$ \\
Obsv. & 35 & 35 & 35 & 39 \\
\hline$R^{2}$ & 0,92 & 0,92 & 0,92 & 0,90 \\
\hline \hline
\end{tabular}

Notas: A variável dependente é o índice de preços das habitações para o Distrito de Lisboa (ver anexo estatístico para mais detalhes); t-estatísticas em parêntesis.

* significativo a $10 \% ; * *$ significativo a $5 \%$

A coluna (1) assume variações de rendimento relativas em vez de variações absolutas - uma abordagem mais consistente com o exposto por Kahneman e Tversky (1981) relativamente à forma como os indivíduos formulam as suas decisões de consumo e de poupança ${ }^{15}$. A coluna (2) substitui o rendimento per capita pelo seu valor logarítmico, uma especificação que tem em conta o facto da literatura considerar, na sua maioria, que a relação entre o rendimento per capita e a procura de habitações não segue uma trajectória linear ${ }^{16}$. A regressão (3) utiliza uma nova série de taxas de juro como alternativa ao uso exclusivo da série fornecida pela Caixa Geral de Depósitos (CGD). Assim, a partir do segundo trimestre de 1996 substituímos a série da CGD pela LISBOR (acrescida de um prémio de risco) ${ }^{17}$. Finalmente, a coluna (4) utiliza dados até 1998 para testar as nossas hipóteses. Nas restantes regressões não usámos dados de 1998 devido às razões já apresentadas na introdução deste artigo. Contudo, uma vez que em 1998 ocorreu um novo ciclo negativo de rendimento (especialmente se medido relativamente à 
média do rendimento durante os quatro trimestres anteriores), considerámos que seria interessante alargar o horizonte temporal dos nossos testes ainda que isso significasse impor alguns pressupostos adicionais no sentido de completar a nossa série ${ }^{18}$. Como foi dito, os resultados para cada uma destas especificações são absolutamente idênticos aos originais.

\section{DISCUSSÃO DOS RESULTADOS}

Na introdução deste artigo tivemos já ocasião de discutir a maior parte das consequências destes resultados para a dinâmica de curto prazo dos preços das habitações. No essencial, elas são consistentes com a literatura existente sobre a eficiência do mercado habitacional, que sugere que os períodos de rápido crescimento dos preços das habitações são justificados, principalmente, pelas expectativas míopes dos investidores relativamente à evolução dos preços, e não pela existência de alterações significativas nos fundamentais do mercado habitacional. Para além disso, as nossas conclusões são consistentes com as de Clayton (1997) que refere que os desvios dos preços das habitações relativamente aos seus valores fundamentais estão relacionados com a fase específica do ciclo imobiliário.

O nosso principal contributo, contudo, é o facto dos nossos resultados ajudarem a explicar a forma como se desencadeia, e adquire relevo, esta irracionalidade dos investidores, mesmo na ausência de alterações relevantes nos fundamentais. De facto, os modelos tradicionais dependem, apesar de tudo, de uma interrupção no comportamento míope ou de uma alteração razoável dos fundamentais no início do processo para justificar um novo ciclo imobiliário. A nossa proposta é que, devido ao fenómeno de aversão às perdas no comportamento dos consumidores, uma simples inversão do ciclo de rendimento pode ser suficiente para despoletar ou travar um rápido crescimento dos preços das habitações, independentemente da dimensão das alterações nos fundamentais. Especificamente, depois de um ciclo negativo de rendimento, logo que o seu valor começa a subir, a pressão negativa sobre a procura habitacional que decorre do fenómeno de aversão às perdas desaparece, o que permite uma subida substancial dos preços das habitações, superior mesmo àquela prevista pelo efeito-standard de rendimento. Este súbito aumento dos preços impulsiona, depois, o comportamento míope em que se baseiam os 
booms habitacionais e que é potenciado pela rigidez da oferta. Pelo contrário, como vimos, quando o rendimento real per capita começa a descer, o fenómeno de aversão às perdas exerce um efeito negativo súbito sobre a procura habitacional e, logo, sobre os preços. A dinâmica que se segue é idêntica à observada durante os booms, embora naturalmente de sentido oposto.

Para ilustrar estas conclusões, simulámos as consequências de uma simples inversão do ciclo de rendimento sobre os preços das habitações. Para o efeito, empregámos os parâmetros estimados nas regressões (1) e (2) e considerámos que os valores estatisticamente não significativos são iguais a zero. Mais ainda, para isolar o efeito assimétrico identificado neste artigo, assumimos que a taxa de juro é constante, o stock habitacional está fixo e a taxa de inflação é igual a zero ${ }^{19}$. Assumimos, ainda, que durante os ciclos de rendimento positivos, o rendimento per capita sobe $0,5 \%$ em cada trimestre, enquanto durante os ciclos negativos desce exactamente o mesmo valor. Consideramos que os ciclos positivos duram entre 8 e 12 trimestres, enquanto os ciclos negativos persistem por 4 a 6 trimestres. A representação gráfica dos nossos resultados é dada na figura 1. A série Preço(RD) representa os valores simulados com base no nosso modelo; quanto à série Preço(PW) representa os valores calculados a partir da especificação de DiPasquale e Wheaton (1996). Os resultados confirmam a volatilidade acrescida a que os preços das habitações estão sujeitos, devido ao fenómeno de aversão às perdas, mesmo na ausência de choques significativos nos fundamentais.

Figura 1

SIMULAÇÃO COMPARATIVA DO MOVIMENTO DOS PREÇOS

DAS HABITAÇÕES PARA OS CASOS DO MODELO REFERENCE-DEPENDENT (RD) E DO MODELO TRADICIONAL (PW) 


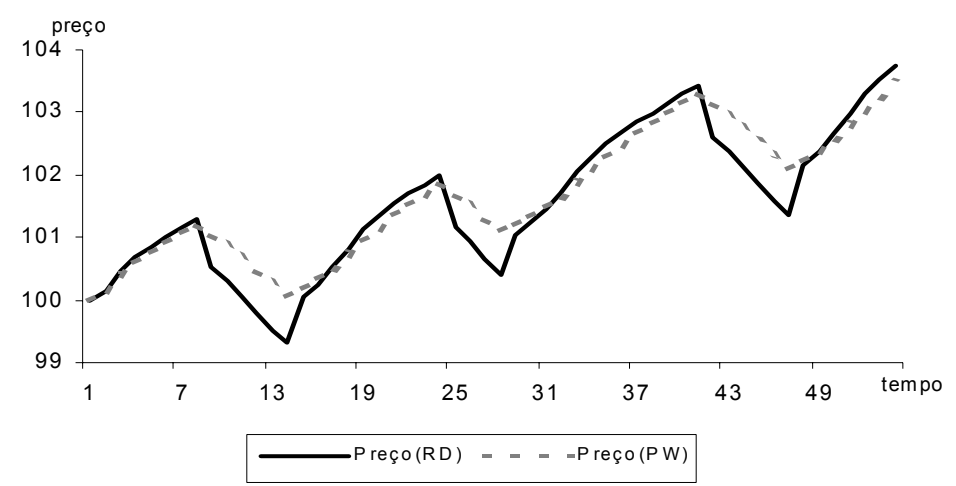

Finalmente, convém abordar uma limitação importante deste artigo, mais precisamente a nossa hipótese, não testada, de que a aversão às perdas é, de facto, uma razão fundamental para a assimetria revelada pelos testes empíricos. Apesar dos resultados serem consistentes com o modelo reference-dependent proposto neste artigo, a insuficiência de dados impede-nos de identificar as fontes precisas das relações estimadas, em particular a importância relativa da aversão às perdas. Existem, no entanto, boas razões, para além dos próprios resultados, para acreditar que a aversão às perdas é de facto uma justificação importante para a assimetria identificada neste estudo.

A primeira razão é a inequívoca influência, já amplamente documentada, da aversão às perdas no comportamento dos consumidores e dos investidores - em particular, nos aspectos assimétricos do mesmo, como inicialmente referido - qual não deverá excluir o mercado habitacional onde, como temos vindo a referir, os aspectos psicológicos assumem reconhecido papel. Exemplo disso mesmo são os resultados de Genesove e Mayer $(2000,2001)$, que identificam a presença de aversão às perdas no mercado imobiliário, embora na perspectiva do vendedor. Evidentemente, por si só isto é insuficiente para avaliar quão importante é o efeito aversão às perdas neste contexto. Por isso, discutimos, a seguir, três possíveis explicações alternativas - aquelas que nos parecem mais relevantes, para além das habituais insuficiências estatísticas: restrições à concessão de crédito hipotecário, custos de transacção e rigidez da oferta.

Relativamente à existência de restrições à concessão de crédito, um argumento possível é que durante os ciclos negativos de rendimento os bancos tenderão a impor condições de empréstimo mais restritivas do que aquelas praticadas durante os ciclos positivos de rendimento. Neste caso, é de esperar que a procura habitacional durante os ciclos negativos 
diminua substancialmente, abaixo mesmo do previsto pelos fundamentais. Prova da importância das restrições à concessão de crédito hipotecário na dinâmica do mercado de habitação é apontada por diversos autores, incluindo Genesove e Mayer $(1997,2001)$. Contudo, estes dois autores referem também que, apesar dessa importância, essas restrições acabam, neste contexto, por ser menos importantes do que a aversão às perdas. De resto, existem pelo menos dois argumentos adicionais que desfavorecem a hipótese das restrições ao crédito. O primeiro, já atrás mencionado, tem a ver com a circunstância da competição entre os bancos, resultante da desregulamentação do sistema financeiro, ter tornado as restrições ao crédito hipotecário em Portugal relativamente pouco sensíveis ao ciclo económico. O segundo, de natureza mais genérica, tem a ver com as conclusões de Shea (1995). O autor conclui que as restrições à concessão de crédito não explicam os padrões (assimétricos) de comportamento dos consumidores em resposta a alterações previsíveis no rendimento. Isto é, o autor não identifica uma relação significativa entre o ciclo económico e o papel das restrições ao crédito nas decisões dos consumidores.

Quanto à segunda explicação possível — custos de transacção - a mesma sugere que a decisão de um indivíduo em comprar uma habitação só se transforma em compra efectiva algum tempo após a decisão ter sido tomada (e como tal depois dos fundamentais justificarem essa decisão). Obviamente, este atraso não existe quando a decisão é não comprar. Assim sendo, enquanto durante um ciclo positivo de rendimento é possível que exista um razoável desfasamento entre a alteração dos fundamentais e o movimento dos preços das habitações, o mesmo não é necessariamente verdade durante os ciclos negativos. Um argumento possível contra esta hipótese é o facto do mercado proceder à imediata actualização dos preços, ao antecipar o crescimento da procura habitacional devido às alterações observadas nos fundamentais e, em particular, no comportamento dos potenciais compradores. Em qualquer caso, elaborámos também um simples teste desta hipótese com base nos nossos dados. Estudámos a possibilidade dos preços das habitações serem uma função contemporânea de alterações negativas no rendimento mas apenas uma função desfasada de alterações positivas. Os resultados, não apresentados aqui, contradizem essa hipótese, revelando de forma consistente a assimetria já identificada nos testes originais, embora se verifique um aumento na robustez da regressão (medida através de $R^{2}$ ) e do coeficiente estimado de $y_{t}$. 
Uma última explicação proposta para justificar a assimetria verificada nos movimentos de curto prazo dos preços das habitações em resposta a alterações no rendimento é a evolução, também assimétrica, do stock habitacional. Enquanto uma subida na procura habitacional corresponderá, em princípio, a um aumento na oferta, quando a procura habitacional cai o stock não é eliminado (a não ser via uma taxa exógena de depreciação). Esta assimetria seria um aspecto tanto mais importante quanto o modelo stock-flow testado assume, implicitamente, um nível fixo de stock habitacional. No entanto, na prática, é improvável que esta seja uma explicação relevante. A razão principal é o facto da resposta da oferta ser normalmente desfasada em relação aos movimentos da procura. Isso implica que, quando a procura habitacional aumenta, o ajustamento é feito prioritariamente via preços, ou seja, um efeito idêntico àquele verificado durante os ciclos negativos de rendimento. A consequência é que qualquer ineficiência que decorre da rigidez da oferta deverá reflectir-se muito mais nas nossas estimativas dos parâmetros tradicionais do modelo stock-flow do que na assimetria decorrente dos ciclos económicos.

Em conclusão, estamos convencidos de que a aversão às perdas é, na verdade, uma razão importante para a assimetria no movimento de curto prazo dos preços das habitações, empiricamente identificada neste estudo. Contudo, não podemos afirmar de forma categórica esta hipótese até a testarmos directamente com base numa análise individual das decisões dos compradores, seguindo a metodologia não agregada adoptada, por exemplo, por Genesove e Mayer (2000, 2001). A falta de dados não nos permite realizar esse teste no presente artigo, mas os nossos resultados sugerem que tal investigação seria interessante, sobretudo na medida em que permitisse uma estimativa mais adequada das verdadeiras implicações quantitativas aqui envolvidas.

\section{CONCLUSÃO}

Os progressos feitos na área da economia comportamental têm contribuído, em muito, para entender alguns dos assuntos em aberto na teoria económica. No caso específico do sector habitacional não seria surpresa que tal também se verificasse, dada a forte componente psicológica observada no comportamento dos intervenientes, à semelhança, por exemplo, do que se constata no sector financeiro. 
Genesove e Mayer $(2000,2001)$ ilustram isso mesmo para o caso da oferta.

$\mathrm{O}$ artigo presente argumenta o mesmo, mas para o lado da procura. Em particular, estudos recentes aplicaram a metodologia comportamental à análise das escolhas dos indivíduos ao nível do consumo e da poupança, sugerindo então que o fenómeno de aversão às perdas — isto é, a relutância exagerada em aceitar perdas - seria responsável pela assimetria exibida nos padrões de consumo dos indivíduos em resposta a boas ou más noticias relativas ao seu rendimento futuro. Contudo, Bowman et al. (1999) concluíram que essa assimetria era menos pronunciada quando avaliado o consumo total e não apenas o consumo (regular) de não duráveis ou serviços. Neste artigo, aproveitamos estes resultados para sugerir que uma explicação possível para os mesmos é o facto de, durante os ciclos negativos de rendimento, os indivíduos compensarem a violação da hipótese do rendimento permanente para bens não duráveis através da diminuição das despesas em bens duráveis. No caso específico do mercado imobiliário, isso significaria uma resposta também fortemente assimétrica da procura habitacional a alterações no nível do rendimento esperado. Enquanto durante ciclos positivos de rendimento os indivíduos tenderiam a manter constante a distribuição do seu consumo entre não duráveis e o bem habitacional - excluindo os tais aspectos de inércia comportamental, o mesmo não aconteceria durante ciclos negativos, durante os quais se assistiria a uma quebra substancial na procura de habitações. O nosso argumento foi, pois, que durante períodos de quebra do rendimento, os movimentos de curto prazo nos preços das habitações exibem uma resposta muito mais forte às alterações do rendimento do que a verificada durante períodos de crescimento do mesmo.

Os resultados dos nossos testes empíricos são consistentes com esta hipótese. Para além disso, confirmam as conclusões apresentadas em estudos anteriores, que sugerem que os booms verificados nos preços das habitações resultam principalmente de expectativas míopes em relação à evolução desses preços, mais do que de alterações significativas nos fundamentais do mercado. O nosso principal contributo é o de propor uma explicação possível para o que despoleta esse comportamento míope, e consequente evolução irracional dos preços, na ausência de alterações significativas nos fundamentais em qualquer fase do processo. De acordo com o nosso argumento, a aversão às perdas no comportamento dos consumidores significa que uma simples inversão do 
ciclo de rendimento pode ser suficiente para iniciar ou terminar um período de rápida expansão dos preços das habitações.

A inexistência de dados apropriados para testar esta hipótese a um nível individual não nos permite assegurar que a assimetria observada é explicada, fundamentalmente, pela aversão às perdas. Justifica-se, pois, a necessidade de mais estudos deste fenómeno a um nível não agregado, não só porque os mesmos permitiriam um conhecimento mais rigoroso das razões e dos parâmetros associados a esta assimetria, mas também porque inferências baseadas em dados agregados não são automaticamente extensíveis a comportamentos e decisões tipicamente individuais.

\section{NOTAS}

${ }^{1} \mathrm{O}$ conceito de inércia comportamental neste contexto refere-se ao facto de os indivíduos ajustarem o seu stock efectivo de bens duráveis apenas quando o diferencial entre o stock efectivo e o desejado atinge um valor razoavelmente alto (isto é, quando existe um choque suficientemente pronunciado que leva a essa discrepância). Uma discussão fundamental deste facto é proporcionada por Caballero (1993). Evidência recente deste comportamento é fornecida por Attanasio (2000).

${ }^{2}$ A conclusão de Bowman et al. (1999) sugere que, pelo menos a um nível agregado, há um ajustamento no consumo de bens duráveis em resposta a uma previsível redução do rendimento que é superior ao ajustamento verificado no consumo de bens não duráveis. A mesma alteração nos padrões de consumo não se verifica no caso em que se espera uma subida no rendimento.

${ }^{3}$ Embora num plano algo diferente, a sugestão de um comportamento assimétrico relativamente às decisões de consumo de bens duráveis não é nova. Attanasio (2000) conclui isso mesmo no caso da compra de automóveis face a alterações dos preços relativos de duráveis e não duráveis.

${ }^{4}$ A série usada para o índice de preços começou a ser recolhida apenas em 1988. Os dados de 1998 foram excluídos do nosso cenário-base em parte porque não nos foi possível recolher dados para todas as variáveis e, também, para evitar distorções induzidas por efeitos da Expo'98. Contudo, dado que em 1998 se assistiu a um novo ciclo negativo de rendimento, acabámos por testar as nossas conclusões principais 
também para o período 1988-1998, assumindo então alguns pressupostos adicionais relativamente às variáveis em falta.

5 Apesar do mercado de crédito hipotecário só ter sido completamente liberalizado no ano de 1991.

${ }^{6}$ Rabin (1998) e Starmer (2000) apresentam dois excelentes levantamentos do conhecimento já existente sobre esta matéria.

${ }^{7}$ A famosa "experiência da caneca", narrada por Kahneman, Knetsch e Thaler (1990), conclui, à semelhança de outros estudos quantitativos, como Galanter e Pliner (1974) e Fishburn e Kochenberger (1979), que o peso que atribuímos a pequenas perdas pode ser duas vezes superior àquele que atribuímos a ganhos equivalentes.

${ }^{8}$ Evidência deste comportamento é fornecida por, entre outros, Herrnstein e Prelec (1992). Estes autores mostram que os indivíduos preferem as alternativas que directamente lhes conferem uma maior utilidade, ignorando as consequências que a sua opção actual possa ter na utilidade de opções futuras - um comportamento que Herrnstein e Prelec definem como resultando de uma abordagem "piecemeal" (em oposição a uma abordagem global). Mais ainda, apesar desta abordagem "piecemeal" ser mais notória quando a decisão em causa é nova, ela acontece também em decisões envolvendo situações repetidas; ou seja, aquelas através das quais mais se poderia aprender acerca dos efeitos de longo prazo das nossas decisões e acerca da nossa função de utilidade global. A um nível mais específico, Loewenstein e Adler (1995) corroboram este resultado. Os autores mostram que os indivíduos tendem a subavaliar a priori o valor de um determinado bem que vão receber no futuro (ou seja, subavaliam o já referido efeito posse). A conclusão é que os indivíduos envolvidos na experiência não ajustaram o seu nível de referência até receberam de facto o objecto, mesmo sabendo que iam receber aquele objecto.

${ }^{9}$ As demonstrações destas proposições, não incluídas neste artigo, podem ser solicitadas aos autores.

${ }^{10}$ Se usássemos uma função de utilidade tradicional do tipo $U\left(c_{t}, h_{t}\right)=c_{t}^{\beta} h_{t}^{1-\beta}$ então $h_{t}=(1-\beta) Y_{t}$ e $\partial h_{t} / \partial Y_{t}$ é constante.

${ }^{11}$ A utilização do número de empregados em substituição do número de famílias pode, na realidade, tornar-se mais apropriada para o estudo em causa. Em primeiro lugar porque o rendimento por trabalhador é provavelmente uma medida mais adequada do ciclo económico. Em segundo lugar, porque a diminuição da dimensão dos agregados familiares no Distrito de Lisboa deverá significar que o aumento verificado no número de famílias sobrestima o verdadeiro aumento qualitativo na procura de habitações. É pois possível que, também neste aspecto, usar o número de trabalhadores seja uma opção preferível. 
12 Temos assim, $U t=i-\frac{1}{4}\left(\frac{P_{t-1}-P_{t-5}}{P_{t-5}}+\frac{P_{t-2}-P_{t-6}}{P_{t-6}}+\frac{P_{t-3}-P_{t-7}}{P_{t-7}}+\frac{P_{t-4}-P_{t-8}}{P_{t-8}}\right)$, onde i é a taxa de juro.

13 Em concreto, assumimos que os indivíduos comparam o seu nível de rendimento presente com a média simples dos rendimentos trimestrais recebidos durante o último ano.

${ }^{14}$ Testes próprios, usando a nossa série, demonstram isso mesmo. Por exemplo, quando repetimos a regressão especificada na coluna (3) incluindo o valor dos preços das habitações do trimestre anterior $\left(P_{t-1}\right)$, verificamos que o coeficiente de persistência trimestral - isto é, o coeficiente de $P_{t-1}$ - ronda os 0,65 . Refira-se, contudo, que as nossas principais conclusões relativamente à dinâmica de curto prazo dos preços das habitações permanecem inalteradas.

${ }^{15}$ Tversky e Kahneman (1981) ilustram este, bastante intuitivo, efeito yardstick com um simples teste (p.457). Eles perguntam a voluntários se estavam dispostos a fazer uma viagem de carro de 20 minutos por forma a poupar $\$ 5$ numa compra de $\$ 15$ e numa compra de $\$ 125$, respectivamente. Enquanto a maioria dos voluntários respondeu positivamente no caso dos $\$ 15$, o oposto foi verdade para a compra de $\$ 125$, apesar da decisão ser efectivamente a mesma: viajar durante 20 minuto ou gastar $\$ 5$ a mais.

16 DiPasquale e Wheaton (1996) estimam que um aumento de $100 \%$ no rendimento tem um impacte no consumo habitacional entre $35 \%$ e $77 \%$, dependendo do valor de outras variáveis (p.127).

${ }^{17}$ Não utilizamos a LISBOR nas restantes regressões pelo facto do ajustamento dos bancos comerciais a este indexante do mercado monetário ter sido relativamente lento. Com efeito, não é bem claro quando e como foi realizada a transição do indexante da CGD (usado nos nossos testes) para a LISBOR. Uma vez que a nossa série só vai até 1997, a utilização do indexante da CGD para todo o período de tempo parece mais razoável, sabendo que o mesmo continuou a traduzir as principais tendências observadas na evolução das taxas de juro.

${ }^{18}$ Como foi previamente explicado numa nota de rodapé, os únicos dados disponíveis para o stock habitacional em 1998 são anuais. Neste caso, assumimos que a distribuição dos valores foi uniforme ao longo do ano.

${ }^{19}$ Claramente, se assumíssemos uma taxa de inflação positiva teríamos um aumento da volatilidade dos preços das habitações determinado por um reforço da importância da motivação especulativa para a procura habitacional. 
Anexo

ANEXO ESTATÍSTICO

As fontes de informação consultadas e o tratamento estatístico preliminar a que os dados foram submetidos são em seguida referenciados.

Índice de preços dos fogos para o Distrito de Lisboa. Os preços trimestrais (preços constantes de 1988) foram obtidos a partir da média dos valores de oferta trimestrais ${ }^{20}$, respeitantes às diferentes zonas consideradas pela Confidencial e Imobiliário, ponderada em função do seu peso no mercado imobiliário do respectivo Distrito.

Stock habitacional para o Distrito de Lisboa. Calculado com base nos dados dos censos de 1991 (INE). Para os anos de 1992 a 1998, adicionou-se sucessivamente o número de fogos construídos trimestralmente $^{21}$. Para os anos de 1988 a 1990, subtraiu-se sucessivamente o número de fogos construídos trimestralmente. Foi assumida uma taxa constante de depreciação.

Custo de posse. Respeita ao diferencial entre a taxa de juro anual nominal praticada pela Caixa Geral de Depósitos para o crédito à habitação e a taxa de valorização média anual nominal, calculada de acordo com o enunciado no texto do artigo. Desprezaram-se os benefícios fiscais em sede de IRS e ainda as bonificações fiscais existentes para segmentos específicos da população ou do mercado, por se manterem relativamente constantes em termos reais.

Emprego. Reporta ao total dos quadros de pessoal das empresas do Distrito de Lisboa em cada trimestre. O valor trimestral foi estimado com base na evolução trimestral do número de empregados por conta de outrem a nível nacional e na evolução anual do número de empregados por conta de outrem a nível distrital.

Rendimento real per capita. Reporta ao PIB trimestral distrital (preços constantes de 1988) estimado com base no PIB nacional trimestral $^{22}$ e no PIB anual regional (NUTS III) ${ }^{23}$.

\section{NOTAS DO ANEXO}

${ }^{20}$ Os valores de oferta trimestrais (em milhares de escudos por metro quadrado de área útil) foram calculados a partir da média aritmética simples dos valores de oferta mensais publicados pela Confidencial e Imobiliário. Estes valores de oferta mensais 
Aversão às perdas no comportamento do consumidor e a dinâmica de curto prazo dos preços das habitações

são determinados a partir da média dos "preços" de oferta praticados pelas empresas de mediação imobiliária que colaboram com a Confidencial e Imobiliário. Para o cálculo desta média não são considerados os valores máximos ou mínimos recebidos, sempre que a sua diferença para o imediatamente inferior ou superior ultrapasse os $50 \%$.

${ }^{21}$ Considerou-se o número total de fogos concluídos por Distrito publicados pelo INE nas Estatísticas da Construção e da Habitação.

${ }^{22}$ Fonte: INE, Contas Nacionais Trimestrais em milhões de contos.

${ }^{23}$ Fonte: INE, Contas Regionais em milhões de contos.

\section{BIBLIOGRAFIA}

ATTANASIO, O. (2000), "Consumer Durables and Inertial Behaviour: Estimation and Aggregation of (S,s) Rules for Automobile Purchases", Review of Economic Studies, 67, 667-696.

BATEMAN, I., A. MUNRO, B. RHODES, C. STARMER e R. SUDGEN (1997), “A Test of the Theory of Reference-Dependent Preferences", Quarterly Journal of Economics, 112(2), 479-505.

BOWMAN, D., D. MINEHART e M. RABIN (1999), "Loss Aversion in a Consumption-Savings Model", Journal of Economic Behavior and Organization, 38(2), 155-78.

BRICKMAN, P. e D. CAMPBELL (1971), "Hedonic Relativism and Planning the Good Society", in Adaptation-level Theory: a symposium, ed. by M. H. Appley, New York.

CABALlERO, R. (1993), "Durable Goods: An Explanation for Their Slow Adjustment”, Journal of Political Economy, 101(2), 351-84.

CASE, K. e R. SHILLER (1988), "The Behavior of Home Buyers in Boom and PostBoom Markets", New England Economic Review, Nov/Dec, 29-46.

CASE, K. e R. SHILLER (1989), "The Efficiency of the Market for Single-Family Homes", American Economic Review, 79(1), 125-37.

CLAYTON, J. (1996), "Rational Expectations, Market Fundamentals and Housing Price Volatility", Real Estate Economics, 24(4), 441-70.

ClAYTON, J. (1997), "Are Housing Price Cycles Driven by Irrational Expectations?", Journal of Real Estate Finance and Economics, 14, 341-63. 
CLAYTON, J. (1998), "Further Evidence on Real Estate Market Efficiency”, Journal of Real Estate Research, 15(1/2), 41-57.

DIPASQUALE, D. e W. WHEATON (1996), "Urban Economics and Real Estate Markets", Prentice Hall, New Jersey.

FISHBURN, P. C. e KOCHENBERGER, G. A. (1979), “Two-piece Von NeumannMorgenstern Utility Functions”, Decision Sciences, 10(4), 503-532.

GALANTER, E. e PLINER, P. (1974), "Cross-Modality Matching of Money Against Other Continua”, in Sensation and Measurement, H. R. Moskowitz et al. (eds.), Reidel Press.

GENESOVE, D. e C. MAYER (1997), "Equity and Time to Sale in the Real Estate Market", American Economic Review, 87(3).

GENESOVE, D. e C. MAYER (2000), "Loss Aversion and Seller Behavior: Evidence from the Housing Market", Wharton School, University of Pennsylvania, mimeo.

GENESOVE, D. e C. MAYER (2001), "Loss Aversion and Seller Behavior: Evidence from the Housing Market", Quarterly Journal of Economics, forthcoming.

HEATH, C. S. HUDDART e M. LANG (1999), "Psychological Factors and Stock Option Exercise", Quarterly Journal of Economics, 114(2), 601-24

HERRNSTEIN. R, e D. PRELEC (1992), "Melioration", in Choice over Time, G. Loewenstein and J. Elster (eds.), Russel Sage Foundation.

KAHNEMAN, D. e A. TVERSKY (1979), "Prospect Theory: an Analysis of Decision Under Risk”, Econometrica, 47(2), 263-291.

KAHNEMAN, D. e A. TVERSKY (1984), "Choices, Values, and Frames”, American Psychologist, 39(4), 341-350.

KAHNEMAN, D., J. KNETSCH, e R. THALER (1990), "Experimental Tests of the Endowment Effect and the Coase Theorem", Journal of Political Economy, 98(6), 1325-48.

LOEWENSTEIN, G. e D. ADLER (1995), "A Bias in the Prediction of Tastes", Economic Journal, 105(431), 929-37.

RABIN, M. (1998), "Psychology and Economics", Journal of Economic Literature, 36(1), 11-46.

ROSEN, K., L. SMITH, e G. FALLIS (1988), "Recent Developments in Economic Models of Housing Markets”, Journal of Economic Literature, 26(1), 29-64.

SHEA, J. (1995), "Union Contracts and the Life-Cycle/Permanent-Income hypothesis", American Economic Review, 85(1), 186-200.

STARMER, C. (2000), "Developments in Non-Expected Utility Theory: The hunt for a descriptive theory of choice under risk", Journal of Economic Literature, $38(2), 332-82$. 
Aversão às perdas no comportamento do consumidor e a dinâmica de curto prazo dos preços das habitações

TVERSKY, A. e D. KAHNEMAN (1981), "The Framing of Decisions and the Psychology of Choice", Science, 211, 453-458.

TVERSKY, A. e D. KAHNEMAN (1991), "Loss Aversion in Riskless Choice: A Reference-Dependent Model", Quarterly Journal of Economics, 106(4), 1039-61.

WHEATON, W. (1999), "Real Estate Cycles: Some Fundamentals", Real Estate Economics, 27(2), 209-230. 
